\title{
Field observation of male-male fighting during a sexual interaction in the whip scorpion Typopeltis stimpsonii (Wood 1862) (Arachnida: Uropygi)
}

\author{
Yuya Watari ${ }^{1 *} \&$ Hirotaka Komine ${ }^{2}$ \\ ${ }^{I}$ Department of Wildlife Biology, Forestry and Forest Products Research Institute, \\ 1 Matsunosato, Tsukuba, Ibaraki 305-8687, Japan \\ E-mail: ywatari@affrc.go.jp \\ ${ }^{2}$ Laboratory of Wildlife Management, Tokyo University of Agriculture and Technology, \\ 3-5-8 Saiwai-cho, Fuchu, Tokyo 183-8509, Japan \\ *Corresponding author
}

\begin{abstract}
We observed male-male fighting during the mating process of the whip scorpion Typopeltis stimpsonii (Wood 1862) in the field on Amami-Oshima Island, Japan, and this may be the first report of agonistic behavior between male whip scorpions. The fight proceeded in the form of physical contact activities, such as wrestling, as the males grappled and tackled each other using their pedipalps and lifted, rotated, and mounted the opponent. Each male held one of the two antenniform legs of the female with his chelicerae throughout the entire fight even during aggressive physical contact. The males fought without chemical spraying, supporting previous studies of the function of chemical spray as a defense against predators. The fight was terminated by ant attacks, probably due to a decreased sensitivity of the males, who concentrated on fighting, to surrounding environmental risks. Additional observations are desirable to promote further research on whip scorpions, as few studies have been conducted in the field.
\end{abstract}

Key words - ant attack, courtship, agonistic interactions, intrasexual competition, vinegaroons, whipscorpions, antenniform legs

\section{Introduction}

Whip scorpions (Uropygi) are one of the orders in the class Arachnida, consisting of 15 genera and 120 species (Harvey 2013). Biological data regarding this group are limited due to the small size of the order and their inconspicuous characteristics, such as a nocturnal habit, dark body color, slow infrequent movement, lack of attraction to bait and light, and lack of ultraviolet fluorescence (Schmidt 2009).

Examples of the subjects included in these few studies include the chemistry of the antipredator spray (Yogi \& Haupt 1977; Itokawa et al. 1981, 1985; Schmidt et al. 2000), life history (Yoshikura 1965; Weygoldt 1971), and courtship and sperm transfer behavior (Weygoldt 1970, 1971, 1978, 1988; Ferreira et al. 2011; Weygoldt \& Huber 2013; Imaizumi \& Karasawa 2015), all of which are characteristic for the unique traits of whip scorpions in the taxonomy and evolution of Arachnida. However, because most of these studies were conducted in the laboratory (but see Imaizumi \& Karasawa 2015), it remains unknown how individuals interact and how the traits of the species are used in the natural environment.

In this study, we report a field observation of male-male agonistic behavior and accompanying incidents during the mating of the whip scorpion Typopeltis stimpsonii (Wood 1862). Because whip scorpion mating is a complex process that includes indirect sperm transfer (Weygoldt 1970, 1971; Schaller 1971) and is time-consuming, such as a mean of 13 $\mathrm{h}$ in Mastigoproctus giganteus (Lucas 1835) (Schmidt 2009) and $24-48 \mathrm{~h}$ in M. brasilianus (C. L. Koch 1843) (Ferreira et al. 2011), its costs and risks in the natural environment may affect reproductive success, survival rate, and evolution (Magnhagen 1991). Therefore, field observations are important in elucidating the fundamental biology of whip scorpions.

\section{Materials and methods}

Typopeltis stimpsonii is endemic to Japan and is naturally distributed on the islands from Kerama Gap to Kyushu Island (Karasawa et al. 2015) (Fig. 1). Many copulating $T$. stimpsonii were observed opportunistically when we conducted a native frog census from a car during the night of September 7-8, 2013, along a forest road at Yamato Village on Amami-Oshima Island, Japan (Fig. 1). Most of the vegetation along the forest road consisted of secondary forest dominated by evergreen broadleaf trees such as Castanopsis sieboldii and Schima liukiuensis. The weather was calm with no heavy rain or strong winds at night. The temperature and relative humidity at 20:30 on September 7 were 


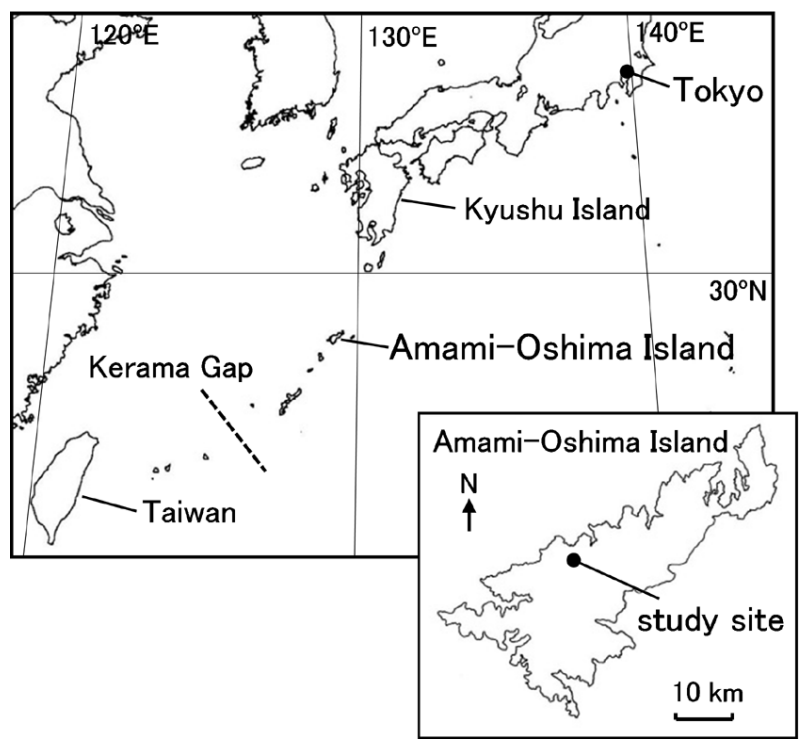

Fig. 1. Location of the study site, Amami-Oshima Island.

\section{$28.2^{\circ} \mathrm{C}$ and $92 \%$, respectively.}

To roughly estimate the maximum frequency of pairs, we set a 50-m long transect using a tape measure along the forest road where the copulating pairs were observed most frequently (N $28^{\circ} 20^{\prime} 33.4^{\prime \prime}$, E $129^{\circ} 21^{\prime} 42.3^{\prime \prime}$ ). Along this transect, individuals occurring on the road (paved and 5-m wide) or within 2-m wide buffers on both sides of the road were counted by two persons (authors) with headlights during a 30 -min walk from $20: 45$ to $21: 15$ on September 7, 2013. The sex of the observed individuals could easily be discriminated by the shape of the patellar apophysis of the pedipalps, i.e., longer, slender, curved in the ventral direction, and tip distinctly broadened in the male and relatively straight and the tip being sharp in the female (Sato 1941; Yoshikura 1966; Haupt \& Song 1996; McMonigle 2013).

Among the pairs we observed that night, we encountered male-male fighting for a female only once ( $28^{\circ} 19^{\prime} 59.0^{\prime \prime}$, E $129^{\circ} 23^{\prime} 48.1^{\prime \prime}$ ) and we made observations with head lights and digital cameras for approximately $90 \mathrm{~min}$ from 0:40 to 2:10 on September 8, 2013, when the fighting stopped. On the basis of the photos and videos taken, we categorized the elements of the fight and summarized their sequence in time throughout the entire fight. It is unlikely that we disturbed the mating individuals because they did not react to our movements or a flashlight, as described by Weygoldt (1970).

\section{Results}

We counted 11 individuals, including five mating pairs and a single female of $T$. stimpsonii along the 50-m transect that we set on the forest road. All pairs observed were at the "stage 2" (a term used by McMonigle 2013) or "generation" (Schmidt 2009) mating phase, in which the male and female were facing the same direction and the female was holding the male's opisthosoma from behind him (Weygoldt 1970,
1971).

Our first encounter with a fighting event appeared to be a parade with three individuals facing the same direction (Fig. 2A). Actually, the front (hereafter, the longer-tailed male) and rear (hereafter, the shorter-tailed male) individuals were males, and the second individual was a female. We could not identify which male paired with the female first because the fight had already begun. Because of their similar body size, it was only possible to visually discriminate between the males based on their tail length. The longertailed male and the female formed the typical reproductive posture of "stage 2" (McMonigle 2013), and the shortertailed male followed the female behind the pair. The longer and shorter-tailed males grasped the tips of the right and left antenniform legs of the female with their chelicerae, respectively. Here we call this formation the "basic formation" (Fig. 2A).

Fighting consists of the basic formation, as when we first encountered the individuals (Fig. 2A), and the ensuing physical contacts, which were repeated several times during the observation (Table 1). The physical contacts included grappling, where the males grappled with each other using their pedipalps (Fig. 2B); mounting, where the offensive male mounted the defensive male and attacked his legs with his palpal chelae or squeezed a leg joint with his pedipalps (Fig. 2C); tackling, where the offensive male tackled the defensive male from below and lifted and turned the defensive male; and pulling the female, where the female completely released the opisthosoma several times during the fight, and the two males pulled each of the female's antenniform legs with their chelicerae (Fig. 2D). The physical contacts were not so strong that the defending male was damaged, cut, or suffered a fatal wound. The physical contacts were most often triggered by the longer-tailed male, which was apparently disturbed when the shorter-tailed male approached the female, and the shorter-tailed male fought back. In most cases, physical contacts started by grappling, followed by any combination of other types of physical contact. The longer-tailed male positioned his opisthosoma close to the female after sequential physical contacts, and the female embraced it again, returning to the basic formation (Fig. 2A). The antenniform legs of the female were held by both males with their chelicerae throughout the entire fight even during aggressive physical contact (Fig. 2).

Ants were also present at this fight. The ants rapidly swarmed the males and female and interrupted the fight. Next, the males and the female tried to remove the ants by shaking their legs rapidly; however, they could not suppress a subsequent aggregation of ants. The interval between physical contacts seemed to increase gradually, and it became difficult for the males to allocate their full concentration to fighting. Finally, the shorter-tailed male released the female's antenniform leg from his chelicerae and abandoned the female as if wanting to escape from the ants rather than from the opponent. Next, the female embraced the opisthosoma of the longer-tailed male, and the pair moved 
A

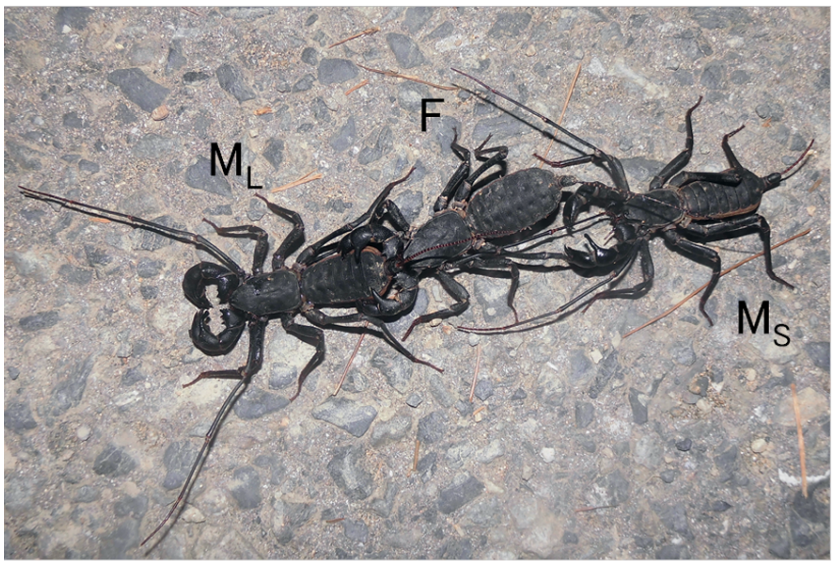

\section{C}

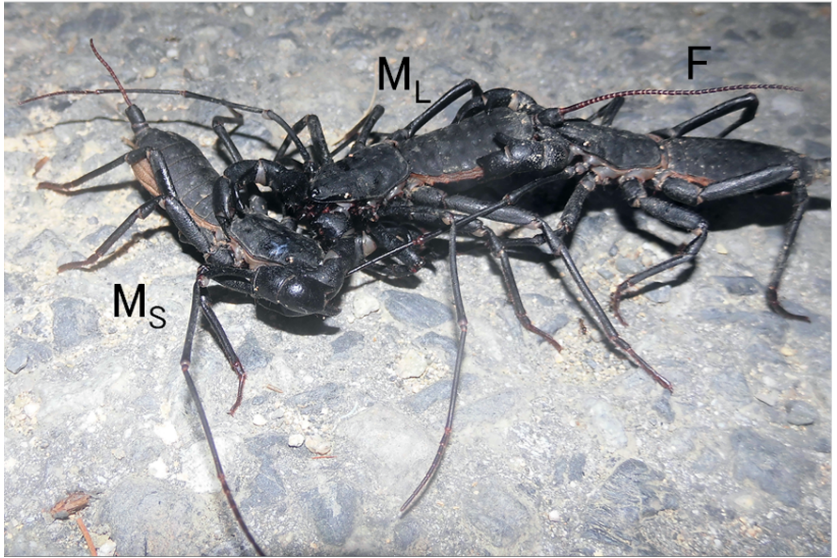

B
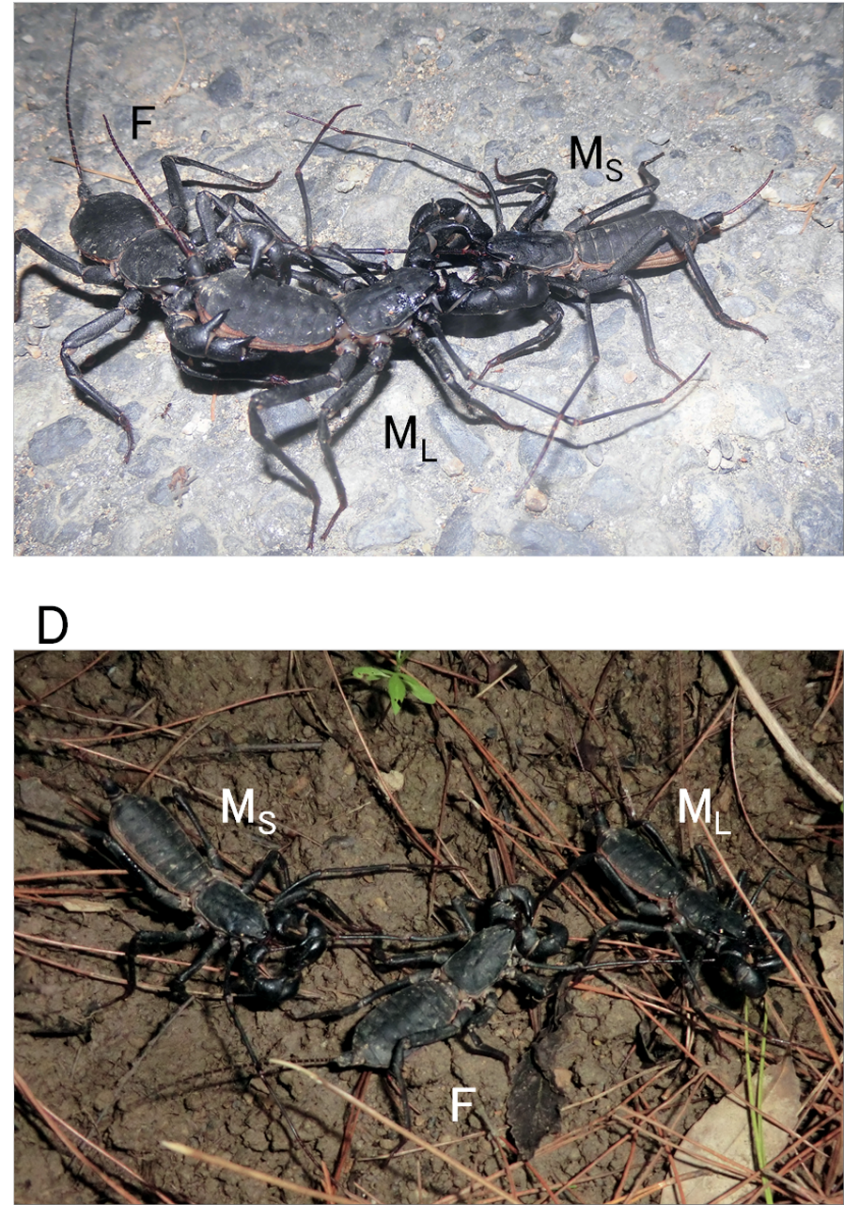

Fig. 2. Elements of male-male fighting of Typopeltis stimpsonii. A, basic formation; B, grappling; C, mounting; D, two males pulled each of the female's antenniform legs with their chelicerae. $M_{L}$ is the male with the long tail (longer-tailed male), $M_{S}$ is the male with a short tail (shorter-tailed male), and $\mathrm{F}$ is the female. The antenniform legs were held by both males throughout the entire fight. Note that the right antenniform leg of the female was held under the body of $\mathrm{M}_{\mathrm{L}}$ by the chelicerae and is hidden in $\mathrm{A}-\mathrm{C}$.

into the bush out of our sight.

Table 1 shows a summary of the time course of the fighting elements and the ant attack every $5 \mathrm{~min}$, based on the photos and videos taken. No single element lasted a full 5 min, except for pulling the female lasting at the time period of 65-. Fighting was conducted on the paved road for the first $35 \mathrm{~min}$, and then continued along the roadside in dense vegetation. Thus, individuals frequently returned to the basic formation after sequential physical contacts in the earlier period, whereas the frequency of basic formation decreased later because the vegetation impeded attempts of a male to smoothly rotate his body and place his opisthosoma in front of a female, thereby making the next physical contact break out without basic formation. Ants also had begun to appear on the photos and videos after the fighting was shifted to the road side. Many ants started to swarm over the bodies of whip scorpions $70 \mathrm{~min}$ after the observations began and the whip scorpions' behavior of shaking their legs rapidly to remove ants was observed more frequently.

\section{Discussion}

This field observation is the first description of malemale agonistic behavior during whip scorpion mating, although male-female and female-female fighting have been reported previously in captivity (Weygoldt 1970). The fight we observed proceeded in the form of the physical contact observed during wrestling, as the males grappled and tackled each other using their pedipalps, and lifted, turned, and mounted their opponent. This observation supports one of the hypotheses of Weygoldt (1988) that sexual dimorphism in the pedipalps, in which the male's pedipalps are longer and stronger than those of the females (Yoshikura 1966; Weygoldt 1970, 1988), helps males with larger pedipalps fight better and allows them to displace another male that has started mating. Moreover, Weygoldt (1988) provided other non-exclusive hypotheses, namely that stronger pedipalps are more successful in manipulating a female during a mating dance, or in performing the final phase of the mating process, named "stage 3" (McMonigle 2013) or 


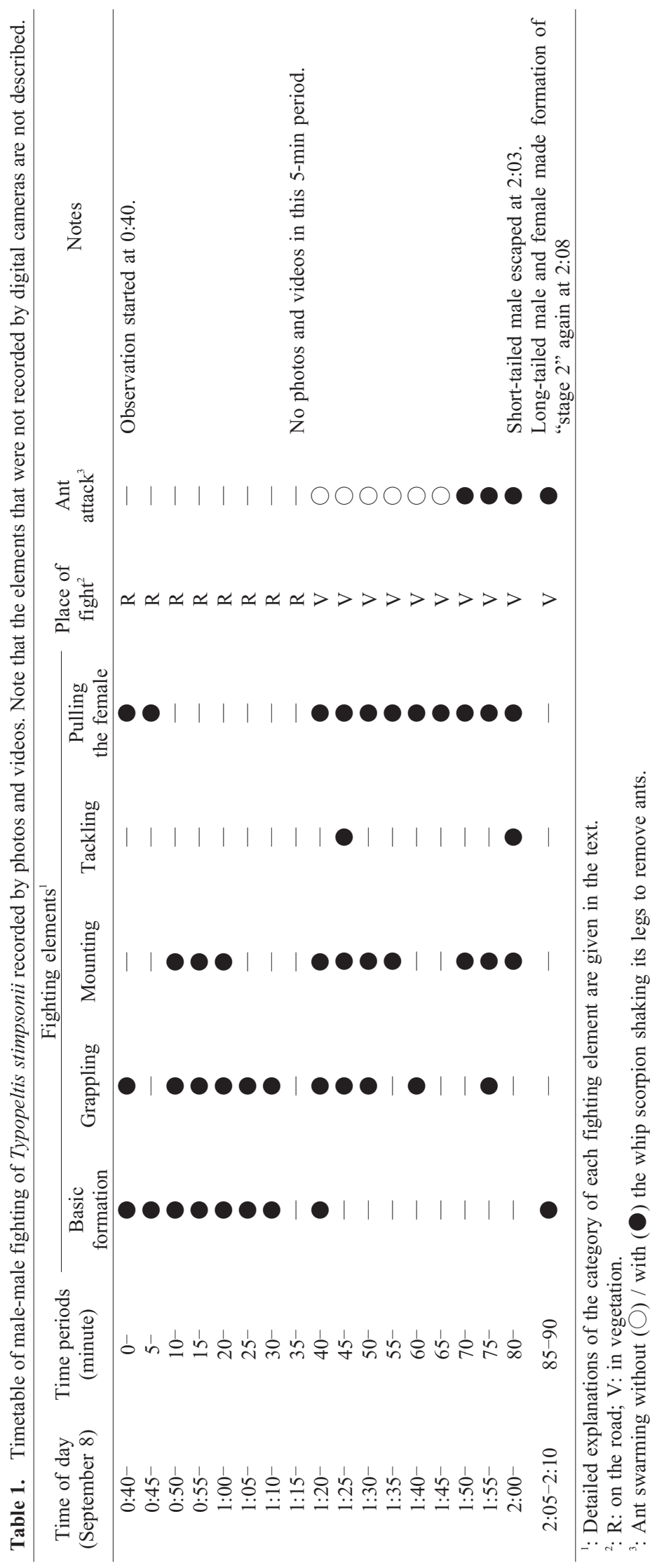


"pressing" (Schmidt 2009), where the male embraces the female's opisthosoma to push sperm packages deep into the female's gonopore. Our observation also supports a previous study about the use of chemical spray. Schmidt et al. (2000) concluded that the chemical spray of the whip scorpions is only used as allomonal defense against predators, based on 4 years of observations. We did not see males fighting with spray, even during aggressive physical contact, which could provide additional support for the chemical spray's function as a defense mechanism against predators.

The interruption of the fight by other organisms (ants in this study) could only be observed in a natural environment. Fighting may reduce the whip scorpions' sensitivity to detecting a surrounding environmental risk. We found that males took a substantial amount of time in examining their opponent's position with their antenniform legs between physical contacts. In addition, because the T. stimpsonii female was held by both of her antenniform legs by the fighting males, which were concentrating and were less aware of their surrounding environment, the female could suffer greater environmental risk during fighting, apart from simply benefiting by acquiring a stronger male. Tradeoffs between costs and benefits in reproduction are likely to be an interesting topic, particularly in whip scorpions, which use their antenniform legs not only as sensors for the surrounding environment (Schmidt 2009) but also for reproductive interaction (Weygoldt 1970; McMonigle 2013; this study). Future research to elucidate the functions of antenniform legs in various situations might provide a clue for evaluating tradeoffs in the reproduction of whip scorpions.

Although we have conducted intensive field surveys for vertebrates and invertebrates on this island at night for many years and have frequently recorded single individuals of $T$. stimpsonii, this was the first time we observed mating of this species with such a high frequency. Therefore, there may be clear peak mating nights within the T. stimpsonii reproductive season. We have not conducted a systematic census of this species and do not have strictly comparable data with unified census methods. However, our records of this species, taken opportunistically in the course of night surveys on this island, do not contradict this expectation (Komine \& Watari unpublished data). For example, during the night of September 6-7, 2013, the day before we observed the fight, we conducted censuses of $13 \mathrm{~km}$ by car and $170 \mathrm{~m}$ by walking and encountered only one pair of mating T. stimpsonii from the car, which was the first time that we observed a mating pair, although the survey site was $20 \mathrm{~km}$ away from that in this study (Amami City). During the night of September 12-13, 2013, when we conducted a 400$\mathrm{m}$ census by walking along the same forest road but at a site different from the 50-m transect set in this study, we did not encounter any individuals. During the night of May 7-8, 2016, we walked along the transect set in this study and observed three individual females. During the night of June 12-13, 2015, we conducted a $2-\mathrm{km}$ census by walking and six single individuals were observed, although the survey site was $10 \mathrm{~km}$ away from that in this study (Amami City). To reveal this seasonal prevalence of reproduction, further research during the reproductive season is warranted.

The biology of whip scorpions is very unique and may provide further insight into the ecology and evolution of Arachnida (Weygoldt 1970). However, as the biology of only a small number of genera has been investigated and few field studies have been conducted, only limited aspects of their biology are available. Thus, our single observation provides important discoveries in whip scorpion biology, including fighting during mating, non-use of the chemical spray during fighting, and mating interrupted by other organisms. Accumulating further observations is desirable for promoting more research on whip scorpions.

\section{Acknowledgments}

We would like to thank Dr. Shigenori Karasawa, Fukuoka University of Education and Dr. Akio Tanikawa, the University of Tokyo for introducing us references. We also thank anonymous two referees for helpful comments.

\section{References}

Ferreira, R. L., Silva, W. D. C., Vieira, V. C. \& Silva, M. S. 2011. Aspects of the behavior and reproduction of Mastigoproctus brasilianus Koch, 1843, (Arachnida: Telyphonidae). Rev. Etol., 10: 3-11.

Harvey, M. S. 2013. Whip scorpions of the World, version 1.0. Western Australian Museum, Perth,

(http://museum.wa.gov.au/catalogues-beta/whip-scorpions).

Haupt, J. \& Song, D. 1996. Revision of East Asian whip scorpions (Arachnida Uropygi Thelyphonida) I. China and Japan. Arthropoda Sel., 5: 43-52.

Imaizumi, A. \& Karasawa, S. 2015. Discovery of Amami whipscorpion Typopeltis stimpsonii (Wood, 1862) in Fukuoka prefecture, Japan. Edaphologia, 96: 19-20. (In Japanese)

Itokawa, H., Kano, R. \& Nakajima, T. 1985. Chemical investitgation of the spray of the Asian whipscorpion Typopeltis stimpsoni (Wood, 1862). Jap. J. Sanit. Zool., 36: 65-66.

Itokawa, H., Kano, R., Nakajima, T., Yasuhara, T. \& Yonabaru, S. 1981. Chemical investigation of the spray of the Asian whip scorpion Typopeltis crucifer Pocock, 1894. Jap. J. Sanit. Zool., 32: 6771.

Karasawa, S., Nagata, S., Aoki, J. I., Yahata, K. \& Honda, M. 2015. Phylogeographic study of whip scorpions (Chelicerata: Arachnida: Thelyphonida) in Japan and Taiwan. Zool. Sci., 32: 352-363.

Magnhagen, C. 1991. Predation risk as a cost of reproduction. Trends Ecol. Evol., 6: 183-185.

McMonigle, O. 2013. Forgotten order of the vinegaroons: whipscorpion biology, husbandry, and natural history. Coachwhip Publ., Greenville, 131 pp.

Sato, I. 1941. Some ecological notes on the whip-scorpion, Typopeltis stimpsoni (Wood). Acta Arachnol., 6: 72-87. (In Japanese)

Schaller, F. 1971. Indirect sperm transfer by soil arthropods. Annu. Rev. Entomol., 16: 407-446.

Schmidt, J. O. 2009. Vinegaroons. Pp. 1038-1041. In: Rech, V. H. \& Cardé, R. T. (eds.) Encyclopedia of Insects. Elsevier, London, $1168 \mathrm{pp}$.

Schmidt, J. O., Dani, F. R., Jones, G. R. \& Morgan, E. D. 2000. Chemistry, ontogeny, and role of pygidial gland secretions of the vinegaroon Mastigoproctus giganteus (Arachnida: Uropygi). J. Insect Physiol., 46: 443-450. 
Weygoldt, P. 1970. Courtship behavior and sperm transfer in the giant whip scorpion, Mastigoproctus giganteus (Lucas) (Uropygi, Thelyphonidae). Behaviour, 36: 1-8.

Weygoldt, P. 1971. Notes on the life history and reproductive biology of giant whip scorpion, Mastigoproctus giganteus (Uropygi, Thelyphonidae) from Florida. J. Zool., 164: 137-147.

Weygoldt, P. 1978. Mating behaviour and spermatophore morphology in whip scorpions: Thelyphonellus amazonicus Butler and Typopeltis crucifer Pocock (Arachnida, Uropygi). Zoomorphologie, 89: $145-156$.

Weygoldt, P. 1988. Sperm transfer and spermatophore morphology in the whip scorpion Thelyphonus linganus (Arachnida: Uropygi: Thelyphonidae). J. Zool., 215: 189-196.

Weygoldt, P. \& Huber, S. 2013. Sperm transfer and maternal care in
Thelyphonus c.f. caudatus from Lombok, Indonesia (Arachnida, Uropygi, Thelyphonida). Zool. Anz., 252: 348-349.

Yogi, S. \& Haupt, J. 1977. Analyse des Wehersekretes bei dem Geibelscorpion Typopeltis crucifer Pocock, 1894. Acta Arachnol., 27: 53-56.

Yoshikura, M. 1965. Postembryonic development of a whipscorpion, Typopeltis stimpsonii (Wood). Kumamoto J. Sci., Ser. B, Sec. 2, 7: 21-51.

Yoshikura, M. 1966. The biology of a whip scorpion, Typopeltis stimpsonii (Wood). Mem. Fac. Gen. Educ., Kumamoto Univ., Ser. Nat. Sci., 1: 31-70. (In Japanese)

Received March 31, 2016 / Accepted May 21, 2016 